\title{
Técnica field in field em planejamentos bidimensionais para irradiação de cérebro total
}

Field in field technique in two-dimensional planning for whole brain irradiation

\author{
A. L. S. Castro ${ }^{1 *}$; T. P. R. Campos ${ }^{1}$ \\ ${ }^{1}$ Departamento de Engenharia Nuclear, Universidade Federal de Minas Gerais, 31270-901, Belo Horizonte-Minas \\ Gerais, Brasil
}

*radioterapia.andre@gmail.com

(Recebido em 18 de abril de 2016; aceito em 01 de julho de 2016)

\begin{abstract}
A radioterapia constitui a modalidade clínica mais utilizada para tratar mestástases cerebrais, tipo mais comum dos tumores secundários provenientes de mama, pulmão e melanomas como origem primária. Os protocolos frequentemente utilizam doses diárias elevadas e, dependendo da técnica de irradiação empregada há elevada probabilidade de complicações em tecidos saudáveis. Com o propósito de minimizar os efeitos adversos, é importante a interpretação dosimétrica do planejamento radioterápico tridimensional por meio de imagens tomográficas ou, no caso de simulações 2D, pelo emprego de técnicas que otimizam a distribuição de dose aumentando a homogeneidade. O estudo objetivou comparar o planejamento $2 \mathrm{D}$ e o conformacional 3D para a irradiação total de cérebro em uma condição individual equivalente e avaliar a evolução desses planejamentos através das técnica field in field. A metodologia consistiu em simular o planejamento bidimensional, reproduzi-lo em um conjunto de imagens tomográficas e compará-lo com o plano conformacional, tanto para técnica de dois campos quanto para a de quatro campos field in field. Os resultados mostraram que não há diferenças significativas entre planejamentos $2 \mathrm{D}$ e $3 \mathrm{D}$ para irradiação de cérebro total, e que a técnica field in field melhorou sensivelmente a distribuição de dose no volume cerebral comparada à técnica de dois campos para a situação proposta. Concluiu-se que um plano bidimensional de quatro campos field in field foi viável na irradiação de cérebro total no tratamento de metástases cerebrais da situação proposta.
\end{abstract}

Palavras-chave: radioterapia, cerebro total, field in field

Radiotherapy is the most used clinical method used for brain metastases treatment, the most frequent secondary tumors provided by breast, lung and melanomas as primary origin. The protocols often use high daily doses and, depending on the irradiation technique there is high probability of complications in health tissues. In order to minimize adverse effects, it is important the dosimetric analysis of three-dimensional radiotherapy planning through tomographic images or, concerning to the 2D simulations, by the application of techniques that optimize dose distribution by increasing the homogeneity. The study aimed to compare the $2 \mathrm{D}$ and $3 \mathrm{D}$ conformal planning for total brain irradiation in a individual equivalent situation and evaluate the progress of these plannings applying the field in field technique. The methodology consisted of simulating a two-dimensional planning, reproduce it on a set of tomographic images and compare it with the conformal plan for two fields and four fields (field in field). The results showed no significant difference between 2D and 3D planning for whole brain irradiation, and the field in field technique significantly improved the dose distribution in brain volume compared with two fields for the proposal situation. As conclusion, the two-dimensional plane for the four fields described was viable for whole brain irradiation in the treatment of brain metastases at the proposal situation.

Keywords: radiotherapy, whole brain, field in field

\section{INTRODUÇÃO}

Metástases cerebrais são as lesões cancerosas malignas mais frequentes do sistema nervoso central. Os cânceres primários mais comuns associados a essas metástases são os de pulmão, mama e melanomas com incidência respectiva variando de $40 \%$ a $50 \%, 15 \%$ a $25 \%$ e $5 \%$ a $20 \%$, respectivamente $[1,2,3]$. As mestástases cerebrais ocorrem, predominantemente, por circulação sanguínea arterial ou venosa e se distribuem proporcionalmente ao fluxo hematogênico no cérebro observando maior incidência nos lobos parietal e frontal [4]. Cerca de $80 \%$ das lesões 
ocorrem nos hemisférios cerebrais, $15 \%$ no cerebelo e 5\% no tronco cerebral [5]. As metástases podem ser solitárias ou se manifestar em múltiplos focos. Series clinicas de diagnóstico por imagem mostraram que $60 \%$ a $70 \%$ de pacientes com metástases cerebrais possuem múltiplas lesões [6] sendo que o melanoma é o tipo de tumor primário mais propenso a originar múltiplas metástases. Os sintomas característicos desses tumores são dores de cabeça, confusão mental e paresia focal, acometendo, aproximadamente, $33 \%$ a $50 \%$ dos pacientes. Os doentes podem apresentar, também, convulsões e ataxia de marcha [7].

As modalidades clinicas, disponíveis atualmente para o tratamento de metástases cerebrais, incluem neurocirurgia, radioterapia e, em alguns casos, quimioterapia. A aplicação de múltiplas modalidades em distintas combinações também constitui uma abordagem comum [8]. Observa-se que a radioterapia permanece como a modalidade clínica preferencial para tratar uma ou múltiplas lesões neoplásicas cerebrais podendo ser usada de forma terapêutica, profilática ou, usualmente, paliativa [3]. Nesse último caso a radioterapia tem sido considerada importante na melhora da qualidade de vida e estabilização das funções neurocognitivas [3,6].

Devido ao fato da maioria dos pacientes com metástases cerebrais possuírem múltiplas lesões, a radioterapia de cérebro total utilizando técnicas bidimensionais (2D) ou tridimensionais (3D) representam as modalidade de tratamento mais aplicadas [6]. Ambas as técnicas podem ser conformacionais, ou seja, diversas geometria de campos, arranjos de feixe e proteções podem ser aplicadas para considerar a posição do volume alvo em relação aos órgãos sadios adjacentes. Essencialmente, a radioterapia 2D tem como principal característica a delimitação do local de tratamento por meio de imagens de raios x ortogonais baseado na localização anatômica de estruturas ósseas. Os algoritmos de cálculo presentes nos sistemas de planejamento de tratamento (TPS) para radioterapia 2D utilizam formalismo e dados triviais considerando o corpo humano homogêneo e constituído de água. Incluem-se correções de contorno, obliquidade de campo, modificadores de feixe e, em alguns casos, correções simples de inomogeneidade de tecidos em que um fator é aplicado para considerar diferenças nas densidades comparativamente à agua [6,9].

Avanços tecnológicos no campo de diagnóstico por imagens permitiram o desenvolvimento de sistemas de planejamento de tratamento que passaram a utilizar imagens de tomografia computadorizada (TC) para representar, volumetricamente, o local de tratamento e a distribuição da dose absorvida. Também foi possível obter informações da densidade eletrônica dos tecidos associadas a números de TC e incorporá-las em algoritmos de cálculo que consideravam de forma mais correta os processos físicos de transferência de energia para o meio [10]. Em adição, a radioterapia 3D possibilitou a avaliação quantitativa do planejamento através da geração de histogramas dose versus volume (DVH) que permitem inferir a porcentagem do volume do tumor, que recebe a isodose de prescrição mínima, e avaliar as probabilidades de complicação do tecido normal dos órgãos de risco, segundo constraints adotados.

Outras técnicas de irradiação modernas também são aplicadas no tratamento de metástases cerebrais. A radiocirurgia estereotaxica tridimensional possibilita a deposição precisa de altas doses com elevado gradiente protegendo o tecido saudável circundante. Essa abordagem é utilizada para controlar tumores inacessíveis cirurgicamente devido à profundidade ou proximidade a órgãos de risco, e também recidivas após a irradiação total do cérebro. Entretanto, a técnica é limitada pelo número e tamanho da lesão, sendo que alguns protocolos mantêm limites de tamanho até $3 \mathrm{~cm}$ de diâmetro e não mais do que três focos de metástases [3,11].

Uma infinidade de protocolos de tratamento foram avaliados em ensaios clínicos prospectivos randomizados de fase III, em pacientes com múltiplas lesões cerebrais submetidos à irradiação de cérebro total [12]. Dos diversos esquemas de fracionamento, $30 \mathrm{~Gy}$ liberados em 10 frações é o protocolo usual [13]. Doses acima de 30 Gy não aumentam a sobrevida ou controle local de pacientes com múltiplas metástases e frações diárias superiores a 3 Gy podem aumentar o risco de toxicidade [14]. Profilaticamente, a irradiação de cérebro total é utilizada para diminuir a incidência de metástases em indivíduos precocemente diagnosticados com câncer de pulmão de pequenas células ou leucemia linfoblástica aguda. Na literatura, encontram-se avaliações de níveis distintos de doses totais de radioterapia na profilaxia das metástases cerebrais [15]. Há consenso que, na radioterapia cerebral profilática, doses elevadas associam-se a uma menor probabilidade de incidência de metástases cerebrais; no entanto, este benefício pouco influenciou no aumento da sobrevida, mas induziu um aumento de toxicidade tardia [16]. A maioria dos 
protocolos de radioterapia profilática adotam doses que variam de 30 a 40Gy, com fracionamento menor ou igual a $2 \mathrm{~Gy} / \mathrm{dia}[17]$.

Os mecanismos de dano cerebral provocados pela radioterapia são complexos e multifatoriais, porém, altamente dependentes da dose total, do fracionamento utilizado e da técnica de tratamento. Classicamente são descritos como devidos à lesão na microvasculatura e a diminuição da população de células gliais [18]. A irradiação cerebral altera o microambiente de células do sistema nervoso central e causa neuro-inflamação, que afeta substancialmente a neurogênese e provocam lesões especificas em células-tronco [3,16]. Para frações de altas doses $(\geq 3 \mathrm{~Gy})$, utilizadas terapeuticamente, ocorre encefalopatia aguda em mais de $50 \%$ dos indivíduos [15]. Os pacientes apresentam especialmente declínio da memória anterógrada e perda da coordenação de movimentos finos, além de inflamações cutâneas e perda de cabelo [18].

Para minimizar a probabilidade de ocorrência de complicações em tecidos saudáveis, justificase o emprego do planejamento conformacional 3D através da reconstituição tridimensional da anatomia do paciente para avaliar e melhorar a distribuição espacial da dose absorvida; ou, em planejamentos 2D, o emprego de técnicas de diminuição de heterogeneidade de dose que reduzem os efeitos adversos da radioterapia. A tabela de procedimentos atual do SUS não prevê o pagamento de radioterapia conformacional 3D para irradiação de cérebro total em metástases cerebrais. Limita-se apenas ao pagamento de planejamento com simulador, ou de acordo com a nova descrição, planejamentos complexos [19].

Em planejamentos 2D simulados, utilizam-se, tipicamente dois campos laterais paralelos e opostos para a irradiação de cérebro total. Um setup clinico padrão usa campos retangulares com o colimador angulado de forma que a borda inferior do campo situe abaixo de uma linha desenhada da glabela do osso frontal ao processo mastoide do osso temporal. As demais arestas do campo devem ultrapassar os limites do crânio [6]. Devido à curvatura do crânio, haverá regiões de altas doses nas extremidades dos ossos frontal, occipital e superior do parietal e essa heterogeneidade é aumentada para fótons de baixa energia podendo gerar efeitos adversos. O uso de campos field in field é uma técnica alternativa aos campos paralelos e opostos convencionais e consiste na adição de outros campos com menor tamanho e peso, a fim de modular de forma simples, a intensidade da radiação e homogeneizar a distribuição de dose. Dessa forma a probabilidade de complicações é reduzida e um tratamento de qualidade superior pode ser conseguido.

Nesse contexto, o trabalho propõe: i) avaliar, para setups padrões, a diferença dosimétrica das modalidades de planejamento 2D e conformacional 3D; ii) comparar os planejamentos 2D field in field e conformacional 3D field in field por meio da distribuição espacial de isodoses, do DVH e de índices de conformidade; e, iii) avaliar a influência de correção de inomogeneidades de tecidos no cálculo da dose absorvida entre planejamento bidimensionais e tridimensionais.

\section{MATERIAL E MÉTODOS}

\subsection{Planejamentos de irradiação de cérebro total com o emprego de dois campos}

O planejamento bidimensional iniciou-se com a aquisição do contorno do crânio de um paciente em um plano axial contendo referências fiduciais utilizando uma régua flexível. As medidas antropométricas foram registradas e o desenho do contorno digitalizado para ser usado no cálculo da distribuição de dose pelo sistema de planejamento CAT3D da Mevis informática médica [20]. Foram definidos, segundo protocolo padrão, dois campos laterais paralelos e opostos, sem blocos de proteção e técnica isocêntrica. A dimensão do campo $17,0 \mathrm{~cm}$ x $14,5 \mathrm{~cm}$ e angulação do colimador de $50^{\circ}$ foram obtidas através de visualização anatômica de radiografia lateral do crânio realizado no acelerador Varian Clinac CL-4 (número de série 287) de energia 4 MV, utilizado também como simulador. O cálculo da dose absorvida foi realizado com o algoritmo collapsed cones convolution sem correção de inomogeneidade.

O mesmo paciente foi submetido a uma TC de crânio com 76 cortes axiais separados por 3 $\mathrm{mm}$ de distância, mantendo as mesmas referências fiduciais demarcadas na simulação 2D. As imagens tomográficas foram inseridas no sistema de planejamento CAT3D e parametrizações 
idênticas às descritas acima, incluindo o algoritmo de cálculo, foram aplicadas, com o intuito de visualizar tridimensionalmente o que foi proposto no planejamento $2 \mathrm{D}$. Logo o chamado "planejamento 3D" nesse trabalho representou simplesmente a reprodução tridimensional de um planejamento com conceitos bidimensionais.

Já o planejamento conformacional 3D (C3D) foi elaborado utilizando o mesmo isocentro dos anteriores, porem com dimensões de campo e quadrado equivalente diferentes devido à adição de blocos de colimação. Os blocos foram conformados utilizando o recurso auto shield do CAT3D com expansão isotrópica em relação ao PTV de $0,5 \mathrm{~cm}$. Nesse plano utilizou-se o algoritmo collapsed cones convolution com correção de inomogeneidade para avaliar de fato a influência das densidades físicas e eletrônicas presentes nos tecidos humanos.

A tabela 1 exibe o resumo dos protocolos dos planejamentos descritos nessa metodologia para o Planejamento 2D, sua reprodução tridimensional, ou seja, o Planejamento 3D e o Planejamento conformacional 3D. Na tabela, QE é a sigla para quadrado equivalente; SSD é a abreviação de source skin distance ou do português distância fonte pele e UM é unidade monitora.

Tabela 1. Protocolos dos planejamentos de 2 campos

\begin{tabular}{ccccccc}
\hline & \multicolumn{2}{c}{ Planejamento 2D } & \multicolumn{2}{c}{ Planejamento 3D } & \multicolumn{2}{c}{$\begin{array}{c}\text { Planejamento } \\
\text { conformacional 3D }\end{array}$} \\
\cline { 2 - 7 } Parâmetros & LD1 & LE1 & LD1 & LE1 & LD1 & LE1 \\
\hline X $(\mathbf{c m})$ & 17,0 & 17,0 & 17,0 & 17,0 & 19,4 & 19,4 \\
Y $(\mathbf{c m})$ & 14,5 & 14,5 & 14,5 & 14,5 & 16,6 & 16,6 \\
QE $\left(\mathbf{c m}^{2}\right)$ & 15,7 & 15,7 & 15,7 & 15,7 & 15,0 & 15,0 \\
Gantry & $270^{\circ}$ & $90^{\circ}$ & $270^{\circ}$ & $90^{\circ}$ & $270^{\circ}$ & $90^{\circ}$ \\
Colimador & $50^{\circ}$ & $310^{\circ}$ & $50^{\circ}$ & $310^{\circ}$ & $50^{\circ}$ & $310^{\circ}$ \\
Mesa & $0^{\circ}$ & $0^{\circ}$ & $0^{\circ}$ & $0^{\circ}$ & $0^{\circ}$ & $0^{\circ}$ \\
Filtro & - & - & - & - & - & - \\
Peso & 0.5 & 0.5 & 0.5 & 0.5 & 0.5 & 0.5 \\
Proteção & - & - & - & - & Sim & Sim \\
SSD $(\mathbf{c m})$ & 72,1 & 72,1 & 71,9 & 72,0 & 71,9 & 72,0 \\
UM & 172 & 172 & 173 & 173 & 181 & 181 \\
\hline
\end{tabular}

2.2 Planejamento otimizado de irradiação de cérebro total usando 4 campos: técnica field in field.

O mesmo contorno axial adquirido na simulação bidimensional do item 2.1 foi utilizado para realizar o Planejamento 2D field in field. Entretanto, ao invés de, executar o plano somente com dois campos paralelos e opostos de $17,0 \mathrm{~cm} \times 14,5 \mathrm{~cm}$, foram adicionados outros dois campos laterais, também, paralelos e opostos, com dimensão reduzida $4,0 \mathrm{~cm}$ x $6,0 \mathrm{~cm}$, e pesos de $10 \%$. As escolhas das dimensões e pesos dos subcampos foram feitas conforme análise de curvas de isodose que minimizavam pontos superiores a $107 \%$ mantendo ainda $100 \%$ da dose prescrita no isocentro do plano. A dimensão " $X$ " do campo foi feita a partir da interpretação dessas curvas no plano axial da imagem bidimensional. Devido à impossibilidade de avaliar a dose no volume, a escolha do tamanho "Y" do campo foi estimada considerando o raio x lateral do planejamento de forma que a dimensão do campo fosse tal que situasse distante das bordas do crânio evitando, assim, possíveis doses elevadas nessas extremidades.

As mesmas parametrizações citadas foram inseridas nas imagens tomográficas do Planejamento 3D field in field e acabaram mostrando-se adequadas. Novamente, a intenção foi reproduzir o planejamento bidimensional em imagens 3D afim de permitir a avaliação quantitativa do plano. $\mathrm{O}$ cálculo realizado não considerou a correção de inomogeneidades de tecidos.

O planejamento conformacional 3D field in field utilizou nos campos de maior dimensão os mesmos blocos de colimação obtidos pela função auto shield descritos no item 2.1. A 
conformação dos subcampos foi realizada com a função draw shield em que os blocos de colimação foram desenhados manualmente protegendo as regiões contendo curvas de isodoses com altos valores. Devido à possibilidade de avaliação da dose em todo o volume do crânio, o peso de $10 \%$ atribuídos aos subcampos e conformações particulares e irregulares minimizaram pontos de dose elevada e mantiveram $100 \%$ da dose prescrita no isocentro. O mesmo número de campos foi mantido para fins de comparação com os demais planejamentos. As distribuições de isodoses e os cálculos das unidades monitoras foram gerados pelo collapsed cones do CAT3D com correção de inomogeneidades.

Tabela 2. Protocolos dos planejamentos de 4 campos field in field.

Planejamento
Planejamento 3D Planejamento
conformacional

\begin{tabular}{ccccccccccccc} 
Parâmetros & LD1 & LD2 & LE1 & LE2 & LD1 & LD2 & LE1 & LE2 & LD1 & LD2 & LE1 & LE2 \\
\hline $\mathbf{X ~ ( c m ) ~}$ & 17,0 & 4,0 & 17,0 & 4,0 & 17,0 & 4,0 & 17,0 & 4,0 & 19,4 & 15,4 & 19,4 & 15,4 \\
Y $(\mathbf{c m})$ & 14,5 & 6,0 & 14,5 & 6,0 & 14,5 & 6,0 & 14,5 & 6,0 & 16,6 & 14,4 & 16,6 & 14,4 \\
Q.E. $\left(\mathbf{c m}^{2}\right)$ & 15,7 & 4,8 & 15,7 & 4,8 & 15,7 & 4,8 & 15,7 & 4,8 & 15,0 & 4,9 & 15,0 & 4,9 \\
Gantry & $270^{\circ}$ & $270^{\circ}$ & $90^{\circ}$ & $90^{\circ}$ & $270^{\circ}$ & $270^{\circ}$ & $90^{\circ}$ & $90^{\circ}$ & $270^{\circ}$ & $270^{\circ}$ & $90^{\circ}$ & $90^{\circ}$ \\
Colimador & $50^{\circ}$ & $50^{\circ}$ & $310^{\circ}$ & $310^{\circ}$ & $50^{\circ}$ & $50^{\circ}$ & $310^{\circ}$ & $310^{\circ}$ & $50^{\circ}$ & $50^{\circ}$ & $310^{\circ}$ & $310^{\circ}$ \\
Mesa & $0^{\circ}$ & $0^{\circ}$ & $0^{\circ}$ & $0^{\circ}$ & $0^{\circ}$ & $0^{\circ}$ & $0^{\circ}$ & $0^{\circ}$ & $0^{\circ}$ & $0^{\circ}$ & $0^{\circ}$ & $0^{\circ}$ \\
Filtro & - & - & - & - & - & - & - & - & - & - & - & - \\
Peso & 0.45 & 0.05 & 0.45 & 0.05 & 0.45 & 0.05 & 0.45 & 0.05 & 0.45 & 0.05 & 0.45 & 0.05 \\
Proteção & - & - & - & - & - & - & - & - & Sim & Sim & Sim & Sim \\
SSD (cm) & 72,1 & 72,1 & 72,1 & 72,1 & 71,9 & 71,9 & 72,0 & 72,0 & 71,9 & 71,9 & 72,0 & 72,0 \\
UM & 155 & 21 & 155 & 21 & 156 & 21 & 156 & 21 & 164 & 21 & 163 & 21 \\
\hline
\end{tabular}

\section{RESULTADOS E DISCUSSÃO}

\subsection{Planejamento de irradiação de cérebro total com o emprego de dois campos.}

A tabela 1 sumarizou os dados dos planejamentos utilizando dois campos. O planejamento 2D foi devidamente reproduzido em 3D, evidenciado pela similaridade dos dados, diferindo minimamente apenas no SSD em $-0,2 \mathrm{~cm}$ (LD1) e $+0,1 \mathrm{~cm}$ (LE1) e no cálculo da unidade monitora que foi $0,6 \%$ superior. Se comparado ao planejamento conformacional 3D verifica-se, obviamente, diferenças no tamanho de campo e no campo quadrado equivalente e, aliado à aplicação de correções de inomogeneidade de tecidos, foi gerado diferenças de 4,5\% no cálculo da unidade monitora. A figura 1 apresenta o beam's eye view do campo Lateral Direito 1 (LD1) para o planejamento 2D, planejamento 3D e conformacional 3D.

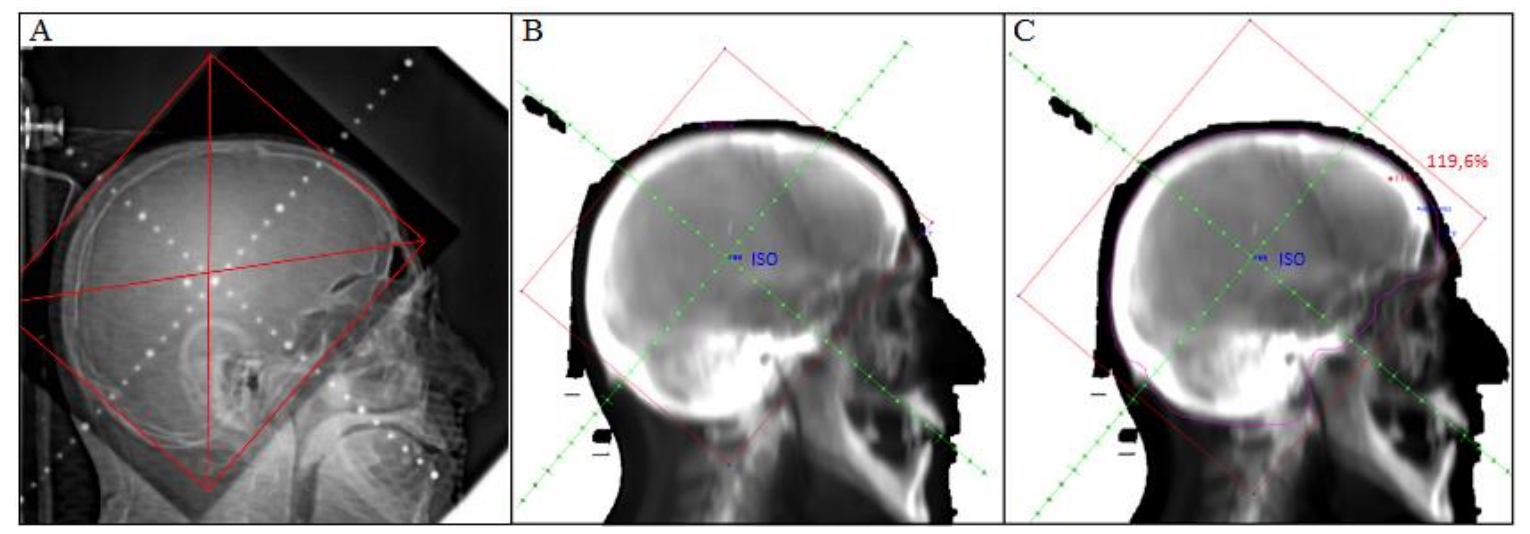

Figura 1: Beam's eye view dos campos LDl do planejamento $2 D(A)$, planejamento $3 D(B) e$ conformacional $3 \mathrm{D}(\mathrm{C})$ onde estão indicados HOT POI-ponto quente e ISO-ponto do isocentro. 
A distribuição espacial das isodoses no plano axial que contém o isocentro (ISO), geradas no planejamento 2D, apresentou isocurvas com topologia equivalente às exibidas no planejamento $3 \mathrm{D}$, conforme esperado, e às exibidas no conformacional 3D, demonstrado na figura 2. Graficamente observou-se regiões de doses superiores a $107 \%$ da dose prescrita em um grande volume do cérebro e doses pontuais muito elevadas nas extremidades dos ossos. Dados quantitativos do histograma dose versus volume do planejamento 2D (reproduzido pelo planejamento 3D) e do conformacional 3D mostraram doses pontuais máximas no volume alvo planejado (PTV) de $120 \%$ e $122 \%$, respectivamente, enquanto que as doses mínimas encontradas no PTV foram, na mesma ordem, $61 \%$ e $87 \%$. A dose mais provável no PTV para os dois planejamentos foi de $112 \%$ da dose prescrita, tabela 3. Apesar da grande inomogeneidade, os histogramas dose volume, figuras $3 \mathrm{~A}$ e $3 \mathrm{~B}$, indicaram cobertura adequada do volume alvo planejado excedendo o parâmetro mínimo V95\% $\geq 95 \%$ [21], ou seja, 95\% ou mais do volume do órgão recebeu $95 \%$ da dose. A qualidade do plano também pode ser avaliada pelo índice de conformidade (IC) que representa a relação entre um volume coberto por uma isodose de referência, adotado nesse trabalho como a isodose de 95\%, e o volume alvo. O planejamento 3D de 2 campos apresentou um IC $=1,43$ e o conformacional 3D teve um IC $=1,31$. Quanto mais próximo esse valor é da unidade mais perfeitamente a isodose de referência conforma o volume alvo. Era esperado, portanto, que um planejamento conformacional apresentasse um melhor índice de conformidade.

As doses avaliadas para órgãos de risco foram os valores pontuais máximos por se tratarem de órgãos em série. Os valores obtidos foram similares, diferindo em maior magnitude apenas na dose máxima absorvida pelo globo ocular, que foi menor no planejamento conformacional 3D. Para valores típicos de irradiação de cérebro total (30 Gy) o cristalino representa o ROI de maior preocupação. Os cristalinos direito e esquerdo receberam doses máximas de 1,8 Gy e 1,5 Gy, em ambos os planos, valores abaixo dos limites de restrição.

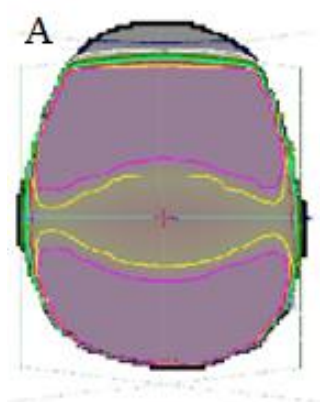

$160 \quad \mathbf{1 7 0}$
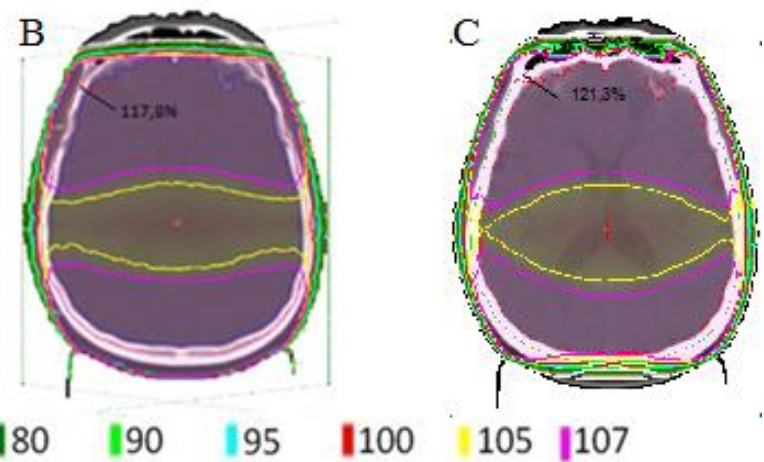

Figura 2: Curvas de isodose no plano axial contendo o isocentro dos planejamento $2 D(A)$, planejamento $3 D(B)$ e conformacional $3 D(C)$ de 2 campos. 


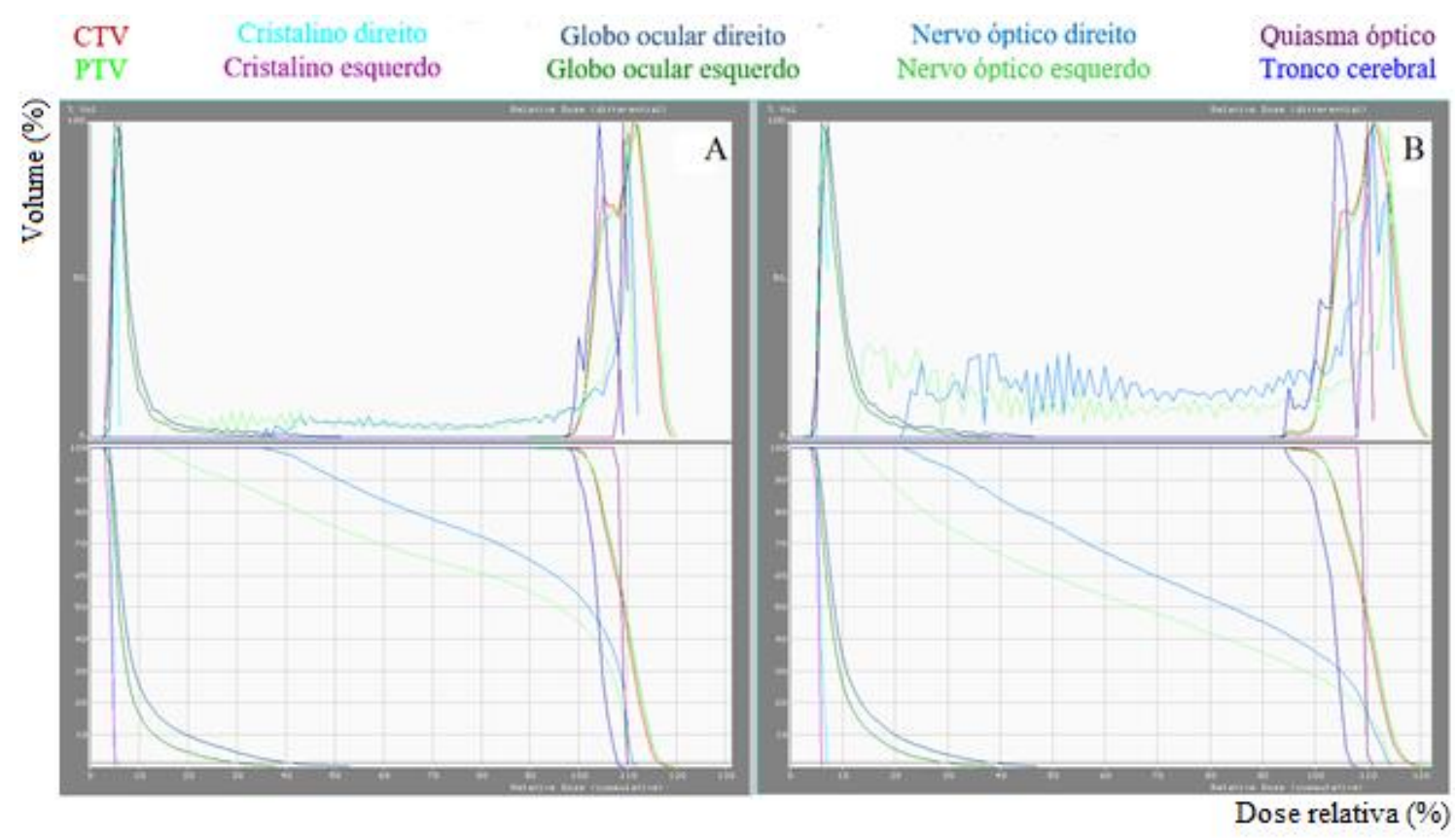

Figura 3: Histograma dose volume dos planejamento $3 D(A)$ e conformacional $3 D(B)$ de 2 campos.

Tabela 3: Relação de volumes em $\mathrm{mm}^{3}$ e valor de porcentagem da dose mínima, máxima, modal e média dos volumes alvo e OARs para planejamento com dois campos.

\begin{tabular}{|c|c|c|c|c|c|c|c|c|c|}
\hline Nome & $\begin{array}{c}\text { Volume } \\
\left(\mathrm{mm}^{3}\right)\end{array}$ & \multicolumn{2}{|c|}{$\begin{array}{c}\text { Dose } \\
\text { mínima (\%) }\end{array}$} & \multicolumn{2}{c|}{$\begin{array}{c}\text { Dose } \\
\text { máxima (\%) }\end{array}$} & \multicolumn{2}{c|}{$\begin{array}{c}\text { Dose } \\
\text { modal (\%) }\end{array}$} & \multicolumn{2}{c|}{$\begin{array}{c}\text { Dose } \\
\text { media (\%) }\end{array}$} \\
\hline Técnica & & 3D & C3D & 3D & C3D & 3D & C3D & 3D & C3D \\
\hline CTV & 1359,0 & 92 & 93 & 119 & 121 & 111 & 111 & 109,2 & 109,5 \\
\hline PTV & 1702,2 & 61 & 87 & 120 & 122 & 112 & 112 & 109,6 & 109,9 \\
\hline Cristalino direito & 0,2 & 4 & 6 & 6 & 7 & 5 & 6 & 5,1 & 6,3 \\
\hline Cristalino esquerdo & 0,2 & 4 & 5 & 5 & 6 & 5 & 6 & 4,7 & 5,9 \\
\hline Globo ocular direito & 7,7 & 3 & 5 & 52 & 47 & 6 & 7 & 10,2 & 11,4 \\
\hline Globo ocular esquerdo & 8,7 & 4 & 2 & 38 & 39 & 5 & 6 & 8,1 & 9,7 \\
\hline Nervo óptico direito & 0,6 & 35 & 22 & 112 & 115 & 110 & 111 & 90,9 & 78,4 \\
\hline Nervo óptico esquerdo & 0,6 & 14 & 13 & 111 & 115 & 110 & 114 & 80,8 & 66,6 \\
\hline Quiasma óptico & 1,7 & 108 & 109 & 110 & 111 & 109 & 110 & 109 & 109,8 \\
\hline Tronco cerebral & 25,5 & 98 & 95 & 109 & 108 & 104 & 104 & 104 & 103,2 \\
\hline Isodose de 95\% - 3D & 2429,7 & & & & & & & & \\
\hline Isodose de 95\% - C3D & 2225,7 & & & & & & & & \\
\hline
\end{tabular}

\subsection{Planejamento otimizado de irradiação de cérebro total usando 4 campos: técnica field in} field.

A fim de homogeneizar a distribuição de dose no plano axial do planejamento $2 \mathrm{D}$ e no volume alvo do planejamento 3D e conformacional 3D foram adicionados subcampos com dimensões e pesos menores, segundo descrito na tabela 2. A reprodução tridimensional do planejamento 2D foi elaborada com equivalência dos parâmetros de entrada na situação em estudo, apresentando apenas diferenças no SSD e no cálculo da unidade monitora. Tais variações atingiram a ordem de $0,6 \%$ e, somente para o campo de maior dimensão. Diferentemente do planejamento $2 \mathrm{D}$ e sua reprodução $3 \mathrm{D}$, em que o crânio foi considerado homogêneo e com a densidade igual à da água, a 
correção de inomogeneidade aplicada no plano conformacional 3D considerou as diferenças de densidade eletrônica e física dos tecidos constituintes, logo o processo de deposição de dose foi alterado influenciando a distribuição espacial de dose e cálculo da unidade monitora. Relativo ao planejamento conformacional 3D, o campo de dimensão maior produziu diferenças no cálculo de aproximadamente 5\% em relação aos demais planejamentos, que pode ser atribuído à aplicação de correção para inomogeneidades e, em uma menor parcela à diferença no campo quadrado equivalente. Já os subcampos assumiram formas irregulares e personalizadas, reproduzindo um campo Q.E. quase idêntico aos demais planejamentos. No entanto, devido ao baixo peso do campo, isso não resultou em uma diferença no cálculo da UM mesmo aplicando a correção de inomogeneidade.

A disposição dos pesos entre os campos maiores e os subcampos é dependente da energia do acelerador, número de campos, dimensão, orientação e geometria dos campos, além da anatomia da região irradiada [9]. No caso estudado em que todas as variáveis foram mantidas fixas para fins de comparação, em um acelerador de $4 \mathrm{MV}$ a distribuição das isodoses considerada pelos autores como ótima foi obtida com atribuição de peso de $10 \%$ para os campos internos. A figura 4 mostra o beam's eye view desses campos, nomeados Lateral Direito 2 (LD2), nos planejamentos 2D, 3D e C3D.

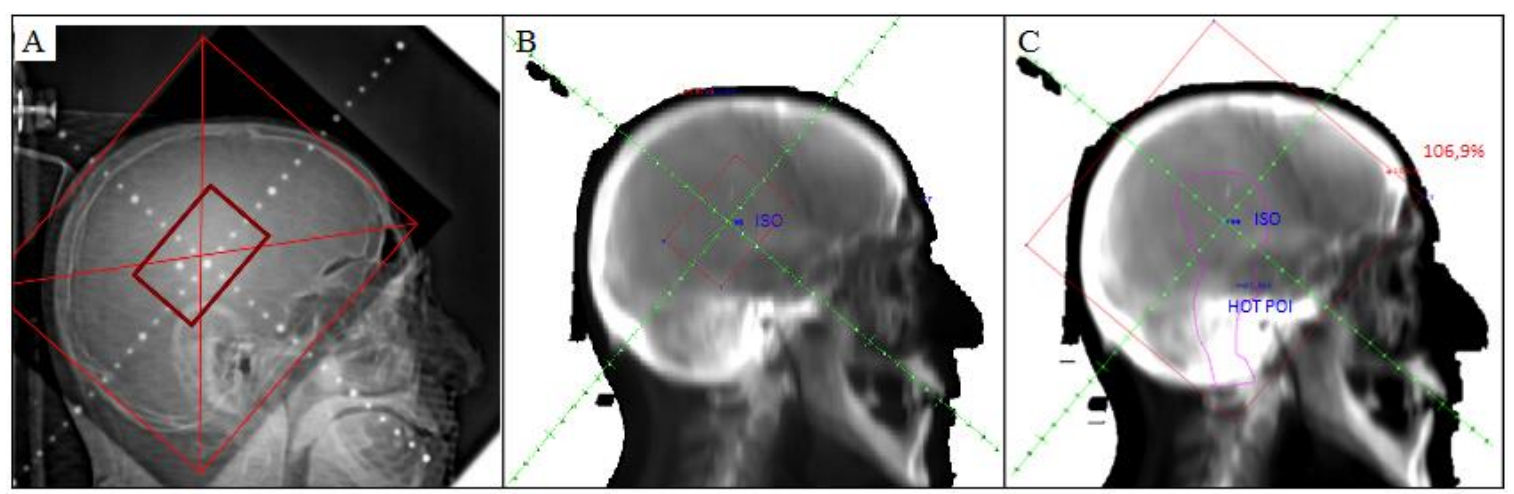

Figura 4: Beam's eye view dos campos LD2 do planejamento $2 D(A)$, planejamento $3 D(B) e$ conformacional $3 D(C)$ onde estão indicados HOT POI-ponto quente e ISSO-ponto do isocentro.

A análise da distribuição das isodoses na imagem axial contendo o isocentro no raio central do campo mostrou semelhanças na topologia das isocurvas referentes aos planos $2 \mathrm{D}$ e sua reprodução 3D com 4 campos. O último, por sua vez, apresentou um número menor de ilhas de isodoses de $107 \%$ comparados ao conformacional 3D (figuras 5B e 5C), no entanto isso não foi observado em todo o volume. Em outros planos axiais que aqui não estão representados observou-se isodoses de valores superiores e uma área relevante de subdosagem (90\%) na região da fossa craniana no planejamento 3D comparado ao C3D.

Segundo o histograma dose volume da reprodução 3D da simulação bidimensional, a dose mais provável no PTV foi de $102 \%$ e, $95 \%$ da dose foi absorvida em $95 \%$ do volume, adequando-se ao limite mínimo considerado aceitável no planejamento, embora a dose pontual mínima encontrada tenha sido de 55\%. O índice de conformidade para a isodose de referência de $95 \%$ foi 1,28. O planejamento conformacional de 4 campos foi mais homogêneo com variação de $112 \%$ a $81 \%$ da dose prescrita. $95 \%$ da dose foi absorvida por $99 \%$ do volume e a dose modal foi de $102 \%$ no PTV. O IC desse planejamento foi igual a 1,2, ou seja, indicou que a isodose de interesse conformou mais adequadamente o volume alvo. Em ambos os planos as doses máximas absorvidas pelos órgãos de risco foram similares, com diferenças sutis nos globos oculares direito e esquerdo. A relação dose volume ou dose máxima em todos os órgãos de risco mantiveram-se abaixo do limite de tolerância, figuras 6A e 6B. 


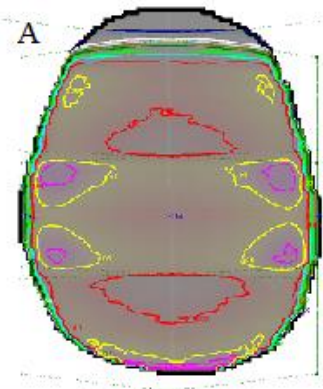

|60 |170
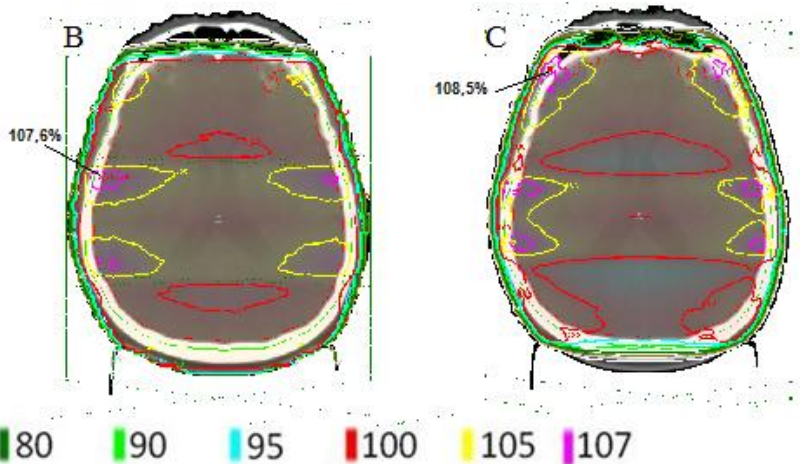

105 |107

Figura 5: Curvas de isodose no plano axial contendo o isocentro (A $e$ B) e DVHs (C e D) dos planejamento $3 \mathrm{D}$ e conformacional $3 \mathrm{D}$ de 4 campos, respectivamente.

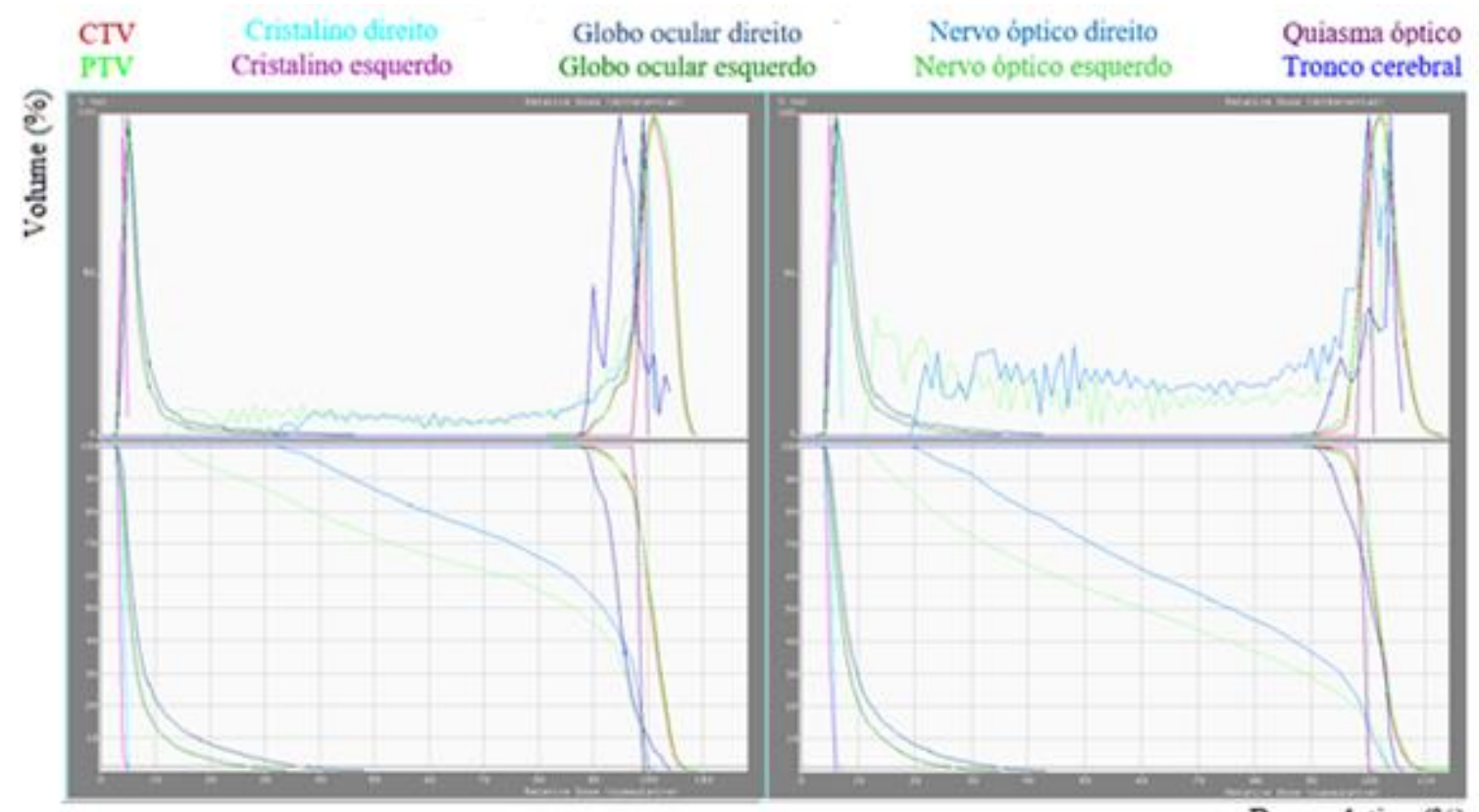

Dose relativa (\%)

Figura 6: Histograma dose volume dos planejamento $3 D(A)$ e conformacional $3 D(B)$ de 4 campos.

Tabela 4: Relação de volumes em $\mathrm{mm}^{3}$ e valor de porcentagem da dose mínima, máxima, modal e média dos volumes alvo e OARs para planejamento com quatro campos field in field.

\begin{tabular}{|c|c|c|c|c|c|c|c|c|c|}
\hline Nome & $\begin{array}{c}\text { Volume } \\
\left(\mathrm{mm}^{3}\right)\end{array}$ & \multicolumn{2}{|c|}{$\begin{array}{c}\text { Dose } \\
\text { mínima (\%) }\end{array}$} & \multicolumn{2}{c|}{$\begin{array}{c}\text { Dose } \\
\text { máxima (\%) }\end{array}$} & \multicolumn{2}{c|}{$\begin{array}{c}\text { Dose } \\
\text { modal (\%) }\end{array}$} & \multicolumn{2}{c|}{$\begin{array}{c}\text { Dose } \\
\text { media (\%) }\end{array}$} \\
\hline & & 3D & C3D & 3D & C3D & 3D & C3D & 3D & C3D \\
\hline CTV & 1359,0 & 83 & 85 & 109 & 113 & 101 & 102 & 100,8 & 102 \\
\hline PTV & 1702,2 & 55 & 79 & 109 & 114 & 101 & 102 & 100,8 & 102,1 \\
\hline Cristalino direito & 0,2 & 4 & 5 & 5 & 7 & 5 & 6 & 4,6 & 5,8 \\
\hline Cristalino esquerdo & 0,2 & 4 & 5 & 5 & 6 & 4 & 5 & 4,1 & 5,3 \\
\hline Globo ocular direito & 7,7 & 4 & 4 & 48 & 43 & 5 & 6 & 9,2 & 10,4 \\
\hline Globo ocular esquerdo & 8,7 & 2 & 2 & 34 & 35 & 5 & 6 & 7,3 & 8,8 \\
\hline Nervo óptico direito & 0,6 & 32 & 20 & 101 & 104 & 99 & 100 & 82,1 & 71 \\
\hline Nervo óptico esquerdo & 0,6 & 12 & 12 & 100 & 104 & 99 & 103 & 72,3 & 60,4 \\
\hline Quiasma óptico & 1,7 & 98 & 99 & 100 & 101 & 99 & 100 & 98,8 & 99,6 \\
\hline Tronco cerebral & 25,5 & 88 & 90 & 104 & 106 & 95 & 104 & 95,5 & 100,5 \\
\hline Isodose de 95\% - 3D & 2172,2 & & & & & & & & \\
\hline Isodose de 95\% - C3D & 2042,7 & & & & & & & & \\
\hline
\end{tabular}


$\mathrm{Na}$ irradiação de cérebro total a interpretação do histograma dose volume e a interpretação gráfica da distribuição espacial de dose indicaram superioridade, mas sem exibir vantagem significativa, do planejamento conformacional 3D sobre o planejamento $2 \mathrm{D}$, independente de se utilizar a técnica de dois campos ou quatro campos field in field. A relação dose-volume no PTV, em todos os planos alcançou a restrição mínima de V95\% $\geq 95 \%$ considerada aceitável para o tratamento em radioterapia. Além disso, as curvas de isodose, assim como as doses absorvidas pelos órgãos de risco apresentaram similares nessas intercomparações. Deve-se ressaltar, porém, que no planejamento bidimensional, reproduzido em 3D, o valor do índice de conformidade foi superior à unidade e superior ao do $\mathrm{C} 3 \mathrm{D}$, significando que um maior volume do crânio além do volume alvo, foi irradiado.

Em relação às técnicas de irradiação de cérebro total de dois campos e de quatro campos field in field, percebe-se que, embora a técnica de quatro campos não tenha reduzido substancialmente a heterogeneidade da dose, avaliada pela diferença da dose máxima e mínima, ela otimizou a distribuição dosimétrica, reduzindo o volume que recebe isodoses elevadas, acima de 107\%, figuras $2 \mathrm{~B}$ e 5B. Salienta-se, contudo, que na técnica field in field o subcampo deve estar localizado na parte central do cérebro e as bordas desse campo devem estar distantes das extremidades ósseas do crânio, como mostra a figura 4A, para que não haja soma das contribuições dos 4 campos e anule o efeito de modulação da intensidade do feixe.

$\mathrm{O}$ índice de conformidade avaliado para os planejamentos 3D ou C3D foram mais próximos da unidade para a técnica field in field, indicativo de melhor qualidade do plano. A dose mais frequente absorvida no volume foi reduzida de $109,6 \%$ para $100,8 \%$ na reprodução do plano 2D, sem comprometimento da relação dose volume no cérebro que se manteve no limite da restrição $\mathrm{V} 95 \% \geq 95 \%$, conforme descrito nos histogramas dose volume, figuras 3A e 6A. O ICRU report 62 sugere que a variação de dose dentro do PTV deva estar restrita entre $95 \%$ e $107 \%$ da dose prescrita, porém, reitera que para casos paliativos pode-se aceitar um maior grau de heterogeneidade da dose. Pequenos volumes recebendo isodoses de $107 \%$ e doses pontuais de $110 \%$ gerados pela técnica de 4 campos não aumentam de forma importante a toxicidade do paciente submetido à irradiação de cérebro total. No entanto, um volume considerável do cérebro recebendo altos valores de dose, encontrados para a técnica de dois campos, pode aumentar a probabilidade de encefalopatia, declínio neuromotor e perda da memória. Isodoses próximas a $120 \%$ nas extremidades do crânio podem provocar inflamações na pele e perda de cabelo. Ademais, o histograma dose volume mostrou que a dose absorvida em todos os órgãos de risco foram superiores no planejamento com dois campos, conforme mostra a tabela 4 .

O sistema único de saúde não contempla o pagamento de TC para metástases cerebrais. Entretanto de acordo com os resultados encontrados na situação de estudo, não houve justificativa clinica ou da física médica que indique um benefício considerável da técnica conformacional 3D para irradiação de cérebro total no tratamento paliativo. Investigação mais aprofundada com maior número de situações clinicas em multicentros é necessária para confirmar os achados. No entanto, para casos profiláticos ou curativos, a radioterapia 3D é a modalidade mais adequada uma vez que ela possibilita utilizar correção para inomogeneidades de tecidos e avaliar mais precisamente a distribuição espacial de dose e cálculo da unidade monitora.

\section{CONCLUSÃO}

A visualização tridimensional da dose absorvida possibilita a inserção de inúmeros subcampos capazes de alcançar um grau de homogeneidade adequadamente elevado. No entanto essa possibilidade não se traduz em planejamentos bidimensionais em que a distribuição espacial de dose é, essencialmente, avaliada em um plano axial, apenas. Isso torna impossível a interpretação dos efeitos referente a várias geometrias e números de campos sob o volume alvo nos planejamentos 2D. Para um planejamento bidimensional utilizando quatro campos field in field os dados quantitativos do estudo selecionado revelaram que o uso dessa técnica reduziu a morbidade do tratamento em relação aos planos de apenas dois campos e promoveu uma distribuição espacial de dose similar às encontradas no planejamento conformacional 3D, para o caso especificado. As diferenças no cálculo da dose absorvida e nas curvas de isodose foram 
atribuídas, principalmente, à maior precisão do planejamento C3D uma vez que, algoritmos de cálculo utilizados em planejamentos tridimensionais possibilitam considerar as influências de inomogeneidades de tecidos. Ademais, no caso em estudo, o plano bidimensional de quatro campos field in field apresentou-se viável em termos de custo efetividade do tratamento e complexidade, e trouxe benefícios compatíveis referente aos planejamentos tridimensionais de irradiação de cérebro total no tratamento de metástases cerebrais.

\section{AGRADECIMENTOS}

Os autores agradecem o suporte financeiro do Conselho Nacional de Desenvolvimento da Pesquisa - CNPq [456719/2013-0 REBRAT-SUS]; do CNPq/PIBIC pela cota de iniciação científica, da Fundação de Pesquisa do Estado de Minas Gerais - FAPEMIG - Universal [FAPEMIG - 18565 FAPEMIG] e Coordenação de Aperfeiçoamento de Pessoal de Nível Superior - CAPES. Os autores agradecem a empresa MEVIS Informática Médica por disponibilizar as ferramentas computacionais ao grupo de pesquisa NRI - Núcleo de Radiações Ionizantes da UFMG.

\section{REFERÊNCIAS BIBLIOGRÁFICAS}

1. Rajan T, Abrey LE. Current management of metastatic brain disease. The Journal of the American Society for Experimental NeuroTherapeutics. 2009 Jul;6:598-603

2. Gazit I, Har-Nof S, Cohen ZR, Zibly Z, Nissim U, Spielgemann R. Radiosurgery for brain metastases and cerebral edema. Journal of Clinical Neuroscience. 2015 22:535-8, doi: 10.1016/j.jocn.2014.08.025.

3. Khan FM. Treatment planning in radiation oncology. Lippincott Williams \& Wilkins; 2007. 527 p.

4. Delattre JY, Krol G, Thaler HT, Posner JB. Distribuiton of Brain Metastases. Arch Neurol. 1988; 45:741.

5. Santos AJ, Franco CMR, Borges LRR, Malheiros SMF, Gabbai AA. Mestástases cerebrais. Rev. Neurociências. 2001; 9(1): 20-6.

6. Levitt SH, Purdy JA, Perez CA, Vijayakumar S. Technical basis of radiation therapy. Berlin: Springer; 2006. 856 p.

7. Posner JB, Chernik NL. Intracranial metastases from systemic câncer. Adv Neurol 1978; 19:579592

8. Sadzikowska JN, Walasek t, Jakubowicz j, Blecharz P, Reinfuss M. Current treatment options of brain metástases and outcomes in patients with malignant melanoma. Reports of pratical oncology and radiotherapy. 2015 Jul 2015: 489-495, doi: 10.1016/j.rpor.2015.12.001

9. Khan FM. The Physics of Radiation Therapy. Lippincott Williams \& Wilkins, ed. 3 Philadelphia. 2003. 560p.

10. Halperin EC, Perez CA, Brady LW. Perez and Brady's Principles and Practice os Radiation Oncology. Lippincott Williams \& Wilkins. 5 ed. 2007. 2050p.

11. Cairncross JG, Kim JH, Posner JB. Radiation therapy for brain metastases. Ann Neurol 1980;7:529-541.

12. Metástases cerebrais múltiplas. Critérios de adequação do ACR. Colégio Brasileiro de Radiologia. 1135-42. Disponível em: </http://www.cbr.org.br//> Acesso em: 27 de fev de 2016.

13. Kurtz JM, Gelber R, Brady LW, Carella RJ, Cooper JS; Radiation Therapy Oncology Group. The palliation of brain metastases in a favorable patient population: a randomized clinical trial by the Radiation Therapy Oncology Group. Int J Radiat Oncol Biol Phys. 1981;7:891- 895.

14. Rades D, Haatanen T, Schild SE. Dose escalation beyond 30 grays in 10 fractions for patients with multiple brain metastases. Cancer 2007;110:1345-1350.

15. Magalhães MS. Radioterapia cerebral profilática no cancro do pulmão de pequenas células extenso [dissertação]. Porto: Instituto de ciências biomédicas Abel Salazar Universidade do Porto; 2010. $26 \mathrm{p}$.

16. Aupérin A, Arriagada R, Pignon JP, et al. Prophylactic cranial irradiation for patients with smallcell lung cancer in complete remission. N Engl J Med. 1999; 341:476-484.

17. Carvalho HA. Radioterapia no câncer de pulmão. J Pneumol 2002 Nov; 28(6): 345-350 
18. Leite MTT, Fonseca APF, Castro ALS. Radioterapia de doenças benignas. Radioterapia Mineira 2015 Out. Disponível em: </http://www.radioterapiamineira.org/.> Acesso em: 16 de fev de 2016.

19. Ministérios da Saúde Secretaria de atenção à saúde . Portaria no 346 , de 23 de junho de 2008.

20. Mevis Informática Médica, Disponível em: <</http://www. mevis.com.br > Acesso: 20 mar, 2016.

21. International commission on radiation units and measurements. Prescribing, recording and reporting photon beam therapy (Supplement to ICRU Report 50). ICRU Report 62. Maryland: 1999. 